JPE 12-2-11

\title{
Active Damping for Wind Power Systems with LCL Filters Using a DFT
}

\author{
June-Seok Lee ${ }^{*}$, Hae-Gwang Jeong ${ }^{*}$, and Kyo-Beum Lee ${ }^{\dagger}$ \\ ${ }^{\dagger *}$ School of Electrical and Computer Engineering, Ajou University, Suwon, Korea
}

\begin{abstract}
This paper proposes a simple active damping algorithm for small-scale wind power systems with an LCL filter. Compared to an L filter or an LC filter, an LCL filter can decrease the harmonics induced by low switching frequencies and produce a satisfactory grid-side current using a comparatively low inductance. Additional active damping of the filter resonance is necessary when an LCL filter is used. This paper introduces an active damping method using a Discrete Fourier Transform (DFT) filter to improve performance without additional sensors or complexity. Experimental results are shown to verify the validity of the proposed algorithm as an active damping method.
\end{abstract}

Key words: Active damping, DFT, LCL filter, Resonance, Wind power system

\section{INTRODUCTION}

Recently, due to global environmental concerns and the gradual depletion of energy resources such as coal and oil, countries around the world have been actively developing wind power systems. Wind turbine technology has been undergoing dramatic development, and wind is now the world's fastest growing energy source [1].

There are two types of wind power generators, the permanent magnet synchronous generator (PMSG) and the doubly fed induction generator (DFIG). The DFIG's structure and control are complicated. Compared to the DFIG, the PMSG has the advantages of the ability to use a wide range of wind speeds and easier control [2]. In small-scale wind power systems, a PMSG, which is connected to the grid through an inverter and boost converter, requires small power conversion devices, which are difficult to design for an LCL filter. In this paper, solutions are proposed to solve these design problems.

As the use of wind power systems has increased, the selection of a suitable inductance has become a significant problem. Finding a practical filter has become even more difficult due to rising costs and poor dynamic responses. These problems can be solved by designing an optimum LCL filter. An LCL filter is a third-order filter that is used with

Manuscript received Aug. 12, 2011; revised Jan. 18, 2012

Recommended for publication by Associate Editor Woo-Jin Choi.

†Corresponding Author: kyl@ajou.ac.kr

Tel: +82-31-219-2376, Fax: +81-31-212-9531, Ajou University

*School of Electrical and Computer Engineering, Ajou University, Korea pulse-width modulation (PWM) rectifiers and an inverter [3], [4]. When it is properly designed, an LCL filter is more appropriate and economical than L or LC filters, because its filter performance is better than that of $\mathrm{L}$ or $\mathrm{LC}$ filters under the same inductance conditions [5]. However, in the design of wind power systems, the LCL filter has a resonance problem [6], [7]. To reduce this resonance problem, this paper presents an active damping algorithm using a Discrete Fourier Transform (DFT).

Recently, research relating to active damping algorithms has attempted to resolve this problem by using an additional resistor and voltage sensors. H. G. Jung proposed PQR power transformations [8], Christian P. Dick presented a digital infinite impulse response filter [9], and Liserre proposed a genetic algorithm [10]. However, these algorithms and methods require either additional voltage sensors or precise tuning of the gains or filter parameters. Furthermore, low-order harmonics are generated by a phase delay due to grid distortion.

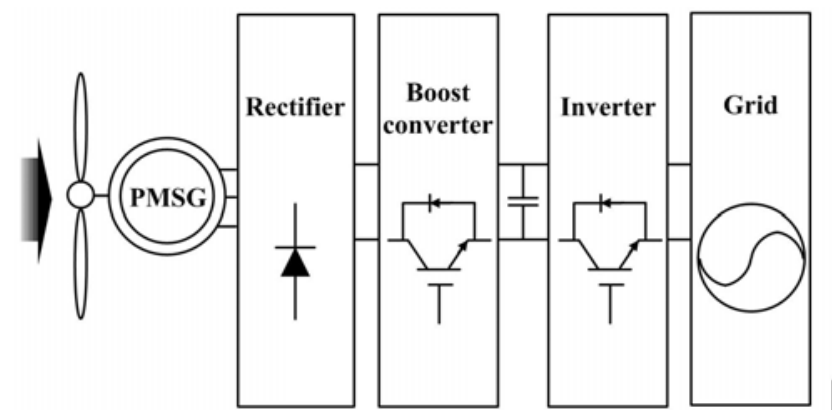

Fig. 1. Grid-connected wind power system. 


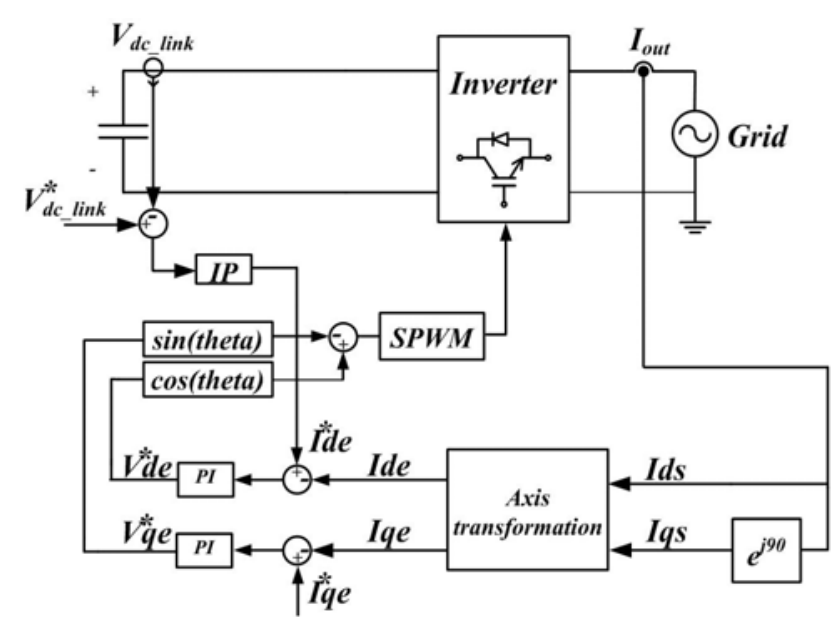

Fig. 2. Block diagram of outer current control method.

In this paper, a DFT is proposed as a method to improve the performance of the resonance compensation. It does not require additional sensors and it is not complicated to apply. When a DFT filter is used, the phase delay is not affected by low-order harmonics, and as a result, this method gives relatively good performance. Experimental results are presented to verify the effectiveness and feasibility of the proposed method.

\section{WIND POWER SYSTEM AND AN LCL FILTER}

\section{A. Wind Power System}

Fig.1 shows the system configuration. This system consists of a PMSG, a three-phase diode rectifier, a boost converter, and an inverter. The PMSG is smaller, lighter, and easier to control than other types of generators. It is selected to improve the generating efficiency and to simplify the control system. The rectifier converts the AC power generated by the PMSG into DC power. One power-switching device is introduced in the boost converter so that maximum power point tracking (MPPT) can be performed. The rectified DC power is boosted by the boost converter, and it is connected to the electric power system through an inverter with a high power factor.

MPPT can be performed at the boost converter [11], [12] or at the inverter [13]. In this system, the boost converter acts as a device for MPPT. The inverter conveys power from the DC-link to the grid. Fig. 2 shows a control block diagram of the grid-connected inverter. The difference between the measured DC-link voltage and the reference DC-link voltage is input to the IP-voltage controller. The difference between the measured current and output (reference current) of the IP-voltage controller is input to the PI-current controller. The output of the PI-current controller is the reference voltage for inverter switching.

\section{B. The LCL Filter Design}

A proper filter deign is very important for improving the quality of the output power in a grid-connected power

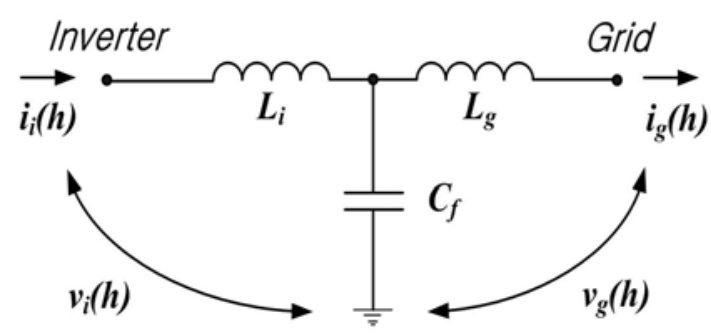

Fig. 3. Equivalent single-phase LCL filter.

converter system. A properly designed LCL filter can reduce the output current harmonics in the bandwidth of the switching frequency. Eq. (1) represents the transfer function:

$$
G(s)=\frac{i(s)}{v(s)}=\frac{1}{L_{T} s} \frac{\left(s^{2}+R_{d} C_{f} z_{L C}^{2} s+z_{L C}^{2}\right)}{s^{2}+R_{d} C_{f} \omega_{r e s}^{2} s+\omega_{r e s}^{2}}
$$

where $z_{L C}^{2}=1 / L_{g} C_{f}, \omega_{\text {res }}^{2}=L_{T} z_{L C}^{2} / L_{g}$, and $L_{T}=L_{i}+L_{g}$.

In Fig. 3, $\mathrm{i}(\mathrm{h})$ and $\mathrm{v}(\mathrm{h})$ indicate the $\mathrm{h}$ harmonic of the current and the voltage. If the damping resistor for the resonance compensation is not connected, Eq. (1) can be expressed as

$$
G(s)=\frac{i(s)}{v(s)}=\frac{1}{L_{T} s} \frac{\left(s^{2}+z_{L C}^{2}\right)}{s^{2}+\omega_{r e s}^{2}} .
$$

When there is no additional damping resistance, the poles and the zeros located on the imaginary axis can generate stability problems due to the resonance. Thus the poles and zeros located on the imaginary axis are moved to ensure stability in the left half of the s-plane by adding a resistor connected to a capacitor.

However, this can cause unnecessary power losses. The power losses can be calculated with an additional resistance as

$$
P_{d}=R_{d} \sum_{h}\left[i_{i}(h)-i_{g}(h)\right]^{2} .
$$

A new active damping scheme using a DFT that does not generate power losses, resonance, or a phase delay is proposed in the next section.

\section{RESONANCE COMPENSATION USING A DFT FILTER}

\section{A. The DFT}

The DFT algorithm can compute both the magnitude and the phase of a signal. The used DFT algorithm has a lower calculation burden than that of the original DFT algorithm [14]-[16], because the calculations of the DFT are applied to a specific frequency. Therefore, a method using the DFT algorithm can compensate the resonance of an LCL filter without any kind of passive damping components.

Eq. (4) and Eq. (5) show the output of the DFT algorithm: 


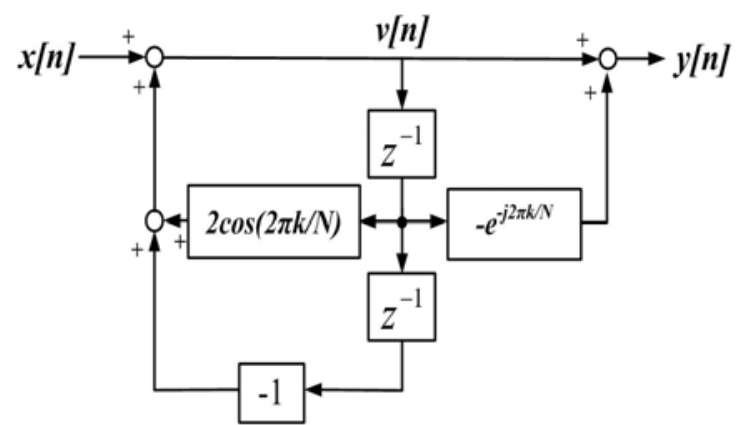

Fig. 4. Block diagram using the DFT algorithm.

$$
\begin{aligned}
& X[k]=\sum_{n=0}^{N-1} X[n] W_{N}^{n k},\left(W_{N}=e^{-j \frac{2 \pi}{N} n k}\right), \\
& H_{G}(z)=\frac{1-e^{-j \frac{2 \pi k}{N}} z^{-1}}{1-2 \cos \left(\frac{2 \pi k}{N}\right) z^{-1}+z^{-2}} .
\end{aligned}
$$

Eq. (4) represents the discrete Fourier function and Eq. (5) shows the z-domain transfer function of the DFT algorithm. When the cyclic frequency is determined to $\mathrm{kf}_{\mathrm{S}} / \mathrm{N}$, the input signals of the DFT resonate at the center of $2 \pi \mathrm{k} / \mathrm{N}$ which is defined to a normalized frequency. Here $f_{S}$ is the signal sampling rate of the DFT algorithm.

The $\mathrm{z}$-domain transfer function $\mathrm{H}_{\mathrm{G}}(\mathrm{z})$ is rearranged in terms of $\mathrm{H}_{1}(\mathrm{z})$ and $\mathrm{H}_{2}(\mathrm{z})$ as

$$
H_{G}(z)=H_{1}(z) \cdot H_{2}(z)=\frac{S(z)}{X(z)} \frac{Y(z)}{S(z)}=\frac{Y(z)}{X(z)} .
$$

Eq. (7) shows the terms of $\mathrm{H}_{1}(\mathrm{z})$ and $\mathrm{H}_{2}(\mathrm{z})$ :

$$
\begin{aligned}
& H_{1}(z)=\frac{S(z)}{X(z)}=\frac{1}{1-2 \cos \left(\frac{2 \pi k}{N}\right) z^{-1}+z^{-2}} \\
& H_{2}(z)=\frac{Y(z)}{S(z)}=1-e^{-j \frac{2 \pi k}{N}} z^{-1}
\end{aligned}
$$

Fig. 4 shows a block diagram of the DFT algorithm. If the frequency of the signal is known, the DFT algorithm can calculate exactly the magnitude and phase of this frequency signal. When the DFT is computed, the magnitude and the phase are

$$
\begin{gathered}
\left|y_{k}(n)\right|^{2}=s^{2}(n-1)+s^{2}(n-2) \\
-s(n-1) \cdot s(n-2) \cdot 2 \cos \left(\frac{2 \pi k}{N}\right) \\
\angle y_{k}[n]=\tan ^{-1}\left\{\frac{\sin \left(\frac{2 \pi k}{N}\right) \cdot s(n-2)}{s(n-1)-s(n-2) \cdot \cos \left(\frac{2 \pi k}{N}\right)}\right\} .
\end{gathered}
$$

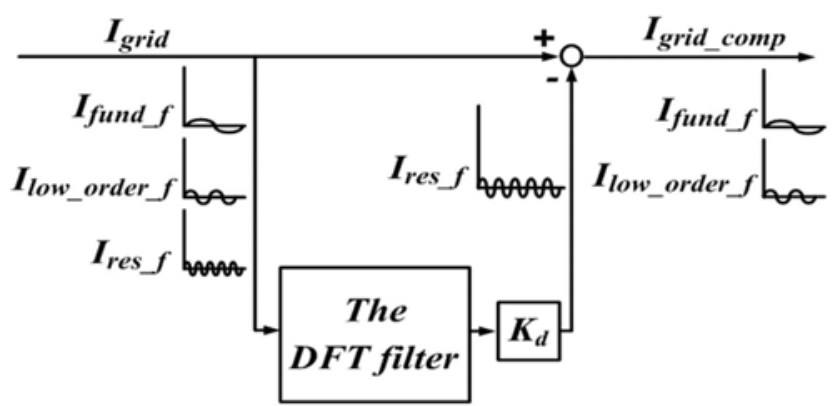

Fig. 5. Block diagram of the proposed active damping method.

In a digital signal processing (DSP) system, the DFT algorithm can extract the signal with a light calculation burden compared with that of the original DFT and the FFT. The DFT algorithm can be implemented in an LCL filter-based grid-connected system as a filter, such as a second filter or an infinite impulse response (IIR) filter, to compensate resonance components due to the LCL filters.

\section{B. Resonance Compensation}

The poles and the zeros introduced by an additional LC part cause resonance in the system because the roots are on the imaginary axis, which can result in a stability problem.

When a system is unstable, the measured grid current has several frequency components, namely, the fundamental component, the low-order frequency component, and the resonant frequency component. This is expressed as:

$$
i_{\text {grid }}=i\left(e^{j \omega_{\text {frnd_freq }}}\right)+i\left(e^{j \omega_{\text {ow_order_freq }}}\right)+i\left(e^{j \omega_{\text {res_freq }}}\right)
$$

To stabilize the system, the DFT is used to extract the resonant frequency components. Using the extracted resonant frequency solves the stability problem. Fig. 5 shows a block diagram of the resonance compensation process.

For this process, Eq. (12) is written as

$$
\begin{aligned}
& \left.i_{\text {grid (fund_freq,low_order_f }} f\right) \\
& \quad=i_{\text {grid (fund_freq,low_order_f,res_f) }}-i_{\text {gird(res_f }} .
\end{aligned}
$$

When using a DFT filter, several elements should be considered for the DFT calculation: the characteristic frequency, the sampling rate, and the buffer size $\mathrm{N}$ (where $\mathrm{N}$ is the number of points).

First, the characteristic frequency is the resonant frequency of the LCL filer in a grid-connected system. The sampling rate and the buffer size are important for extracting the precise resonant frequency.

Second, the sampling rate is determined by considering the characteristic frequency. The sampling period should be shorter than the period of the signal including the characteristic frequency in order to extract it properly. When the sampling 


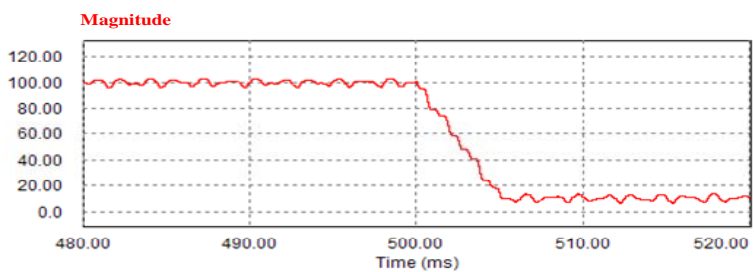

(a)

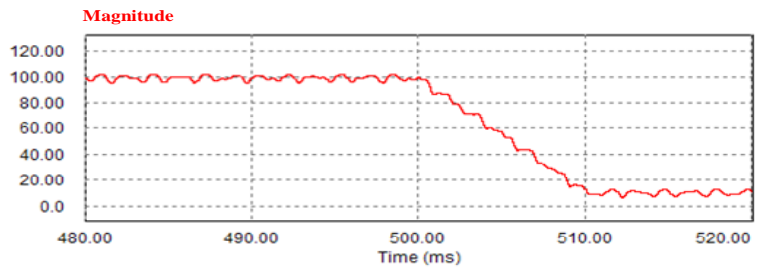

(b)

Fig. 6. Waveform of the DFT with a magnitude change at 500 [ms] for (a) $\mathrm{N}=50$ and (b) $\mathrm{N}=100$.

period of the DFT is longer than the period of the signal including the characteristic frequency, the DFT algorithm cannot make a signal including characteristic frequency. In practice the period of the signal including the characteristic frequency needs to be at least two-time longer than the sampling period.

Third, in choosing the buffer size $\mathrm{N}$, there is a trade-off between the accuracy of the DFT in the steady state and the dynamic response of the DFT. The $y(n)$ of the DFT is computed by using the $s(n)$, which is calculated with the previous values. Depending on the buffer size, the DFT can be used to detect the characteristic frequency more accurately. A high $\mathrm{N}$ means that $\mathrm{y}(\mathrm{n})$ and $\mathrm{s}(\mathrm{n})$ take a long time to change. When a magnitude change occurs, the new sample is added to compute the new $y(n)$ value. However, the rest of the $y(n)$ values have the previous magnitude of the frequency. The change in the magnitude of the frequency is taken into account slowly. On the other hand, a short $\mathrm{N}$ means that a change in the magnitude of the frequency is detected quickly. For example, suppose that the needed frequency is $800[\mathrm{~Hz}]$, which has a magnitude of 100 . Then suppose there is a change in the magnitude to 10 . If the buffer size is 100 , the DFT detects the change in the magnitude in 10 [ms]. However, if the buffer size is 50 , the DFT detects the change in the magnitude in $5[\mathrm{~ms}]$. Fig. 6 shows effect of $\mathrm{N}$.

When the buffer size is long, a DFT is used to compensate the resonant frequency as in Eq. (13), and the compensated $i_{\text {grid }}$ is applied to the current for control. Then, the next magnitude of the resonant frequency is detected in the new measured $i_{\text {grid }}$. Although the new measured value is applied to the DFT, the DFT still computes the previous magnitude. This is due to the fact that the buffer value from 0 to $\mathrm{n}-1$ for a large buffer size is the previous magnitude. The subtraction of the previous magnitude in Eq. (13) makes the reference current more resonant than the uncompensated reference current. Therefore,

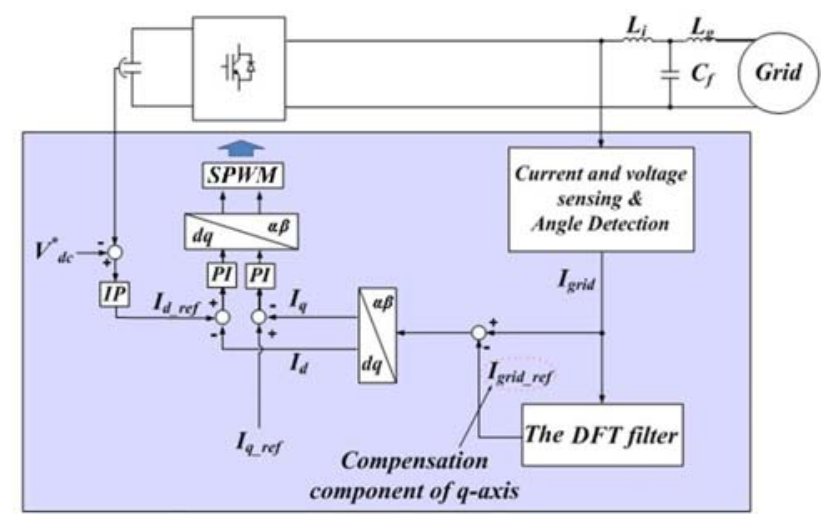

Fig. 7. Resonance compensation block diagram using DFT filter.

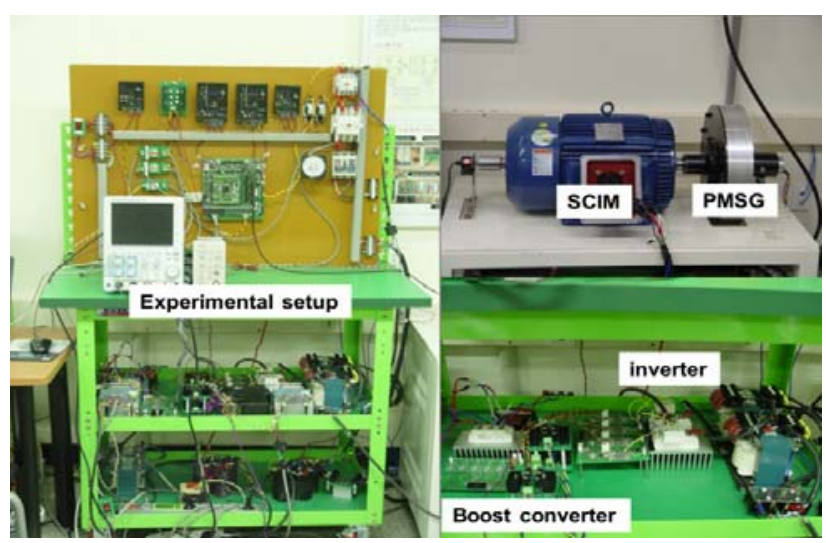

Fig. 8. Experimental setup for wind power system.

choosing a suitable $\mathrm{N}$ is important. The effect of the detection delay in the DFT is minimized by using a gain $\mathrm{K}_{d}$. The used $\mathrm{K}_{\mathrm{d}}$ is smaller than 1. This is because the extracted resonant component cannot be bigger than the real resonant component. If a bigger component than the real resonant component is compensated, the system might be unstable.

Three decisions are necessary for obtaining a good ability to detect the frequency using the DFT algorithm. The choice of the buffer size $\mathrm{N}$ is especially important, because there is a tradeoff between the accuracy of the DFT in the steady state and its dynamic response. Therefore, the sampling rate and the buffer size $\mathrm{N}$ determine the performance of the DFT algorithm.

The three elements determined for the DFT are computed beforehand and saved in the RAM and ROM spaces. Therefore, the DFT filter presents a good alternative to the original DFT and the FFT. It does not require additional sensors and is easily applied with simple control. The phase delay is not affected by the low-order harmonics, and the method has excellent performance. In Fig. 7, the total block diagram is shown.

\section{EXPERIMENTAL RESULTS}

Experiments were performed on a $1.5[\mathrm{KW}]$ PMSG wind power turbine to verify the validity of the proposed method. The experimental setup is shown in Fig. 8. 
TABLE I

SIMULATION PARAMETERS OF THE 1.5 [KW] WIND SYSTEM

\begin{tabular}{|c|c|}
\hline Rated output power & $1500[\mathrm{~W}]$ \\
\hline Grid line-to-line voltage & $220\left[\mathrm{~V}_{\mathrm{rms}}\right]$ \\
\hline Grid frequency & $60[\mathrm{~Hz}]$ \\
\hline DC-link voltage & $350[\mathrm{~V}]$ \\
\hline Rated Current & $9.6\left[\mathrm{~A}_{\mathrm{rms}}\right]$ \\
\hline Switching frequency & $5000[\mathrm{~Hz}]$ \\
\hline Sampling frequency & $5000[\mathrm{~Hz}]$ \\
\hline
\end{tabular}

TABLE II

DESIGN SPECIFICATIONS OF LCL FILTERS FOR 1.5 [KW] WIND SYSTEM

\begin{tabular}{|c|c|}
\hline Grid-side inductance & $4.5[\mathrm{mH}]$ \\
\hline Inverter-side inductance & $9.5[\mathrm{mH}]$ \\
\hline Capacitor (delta connection) & $5[\mu \mathrm{F}]$ \\
\hline Resonant frequency & $1151[\mathrm{~Hz}]$ \\
\hline
\end{tabular}

TABLE III

DESIGN SPECIFICATIONS OF THE DFT FOR 1.5 [KW] WIND SYSTEM

\begin{tabular}{|c|c|}
\hline Sampling rate & $100[\mu \mathrm{s}]$ \\
\hline Number of samples & 85 \\
\hline Normal frequency & $1151[\mathrm{~Hz}]$ \\
\hline
\end{tabular}

The PMSG is coupled with a squirrel-cage induction motor (SCIM). The parameters of the energy storage device and the DFT filter are shown in Tables I-III. The grid voltage is 220 [V]. The DC-link voltage is maintained at $350[\mathrm{~V}]$ in the PWM inverter, which has a switching frequency of $5[\mathrm{kHz}]$. The value of $\mathrm{C}$ of the LCL filter is set to be small to make power factor close to 1 .

Fig. 9 shows the output grid current and the FFT of the output grid current when the system is unstable because of resonance, with a passive damping in which the resistance is connected with a capacitor in series. The output current does not have the resonance, and the magnitude of the resonant frequency is not found in the FFT of the output current. The system becomes stable with passive damping. Because the grid voltage has the 3th, 5th, and 7th order harmonics, the output current is not precisely a sinusoidal wave. However, there is a power loss due to the resistance.

Fig. 10 shows the output grid currents and the FFT of the output current without any damping. The output currents have a ripple. The frequency of the ripple is identified through the FFT of the output current as $1151[\mathrm{~Hz}]$. This frequency is the same as the resonant frequency of the designed LCL filter.

Fig. 11 presents the output of the DFT filter with the parameters from Table III. Fig. 11(a) shows the extracted resonant signal. The frequency of the signal identified through

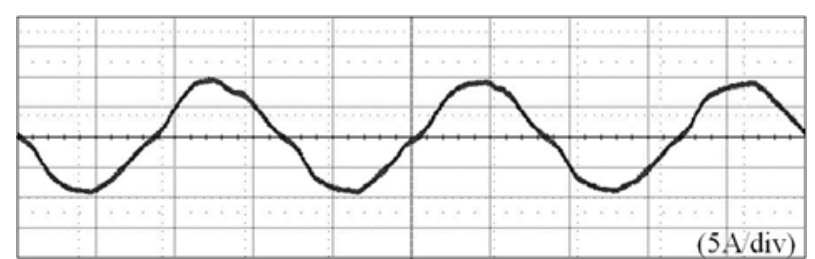

(a) $T / \operatorname{div}=5[\mathrm{~ms}]$.

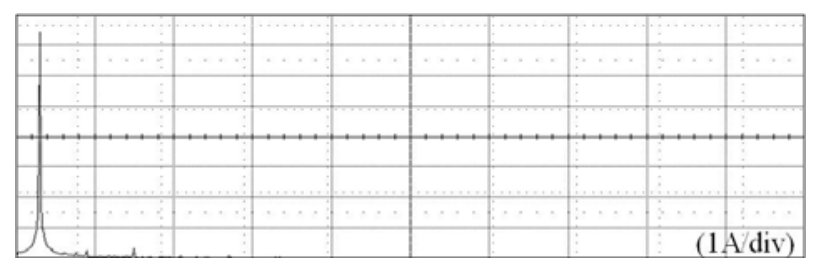

(b) $f / \operatorname{div}=200[\mathrm{~Hz}]$.

Fig. 9. Waveforms of (a) output current and (b) FFT of output current with passive damping.

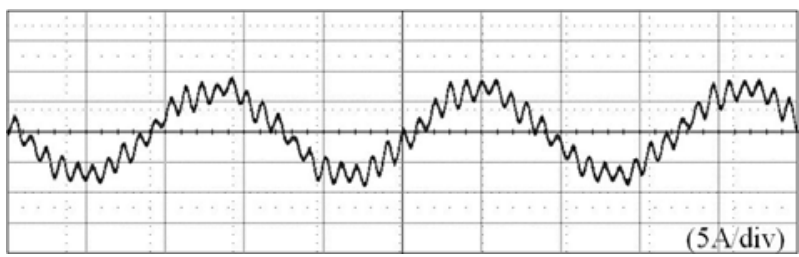

(a) $T / \mathrm{div}=5 \mathrm{~ms}$.

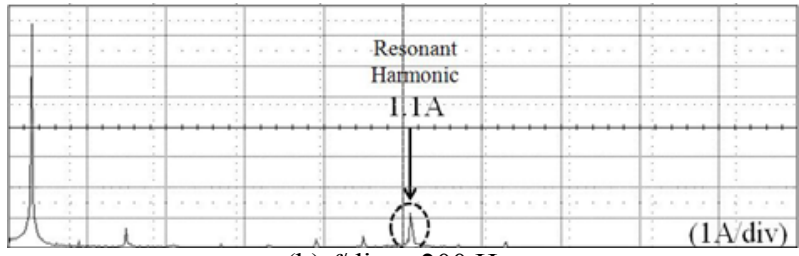

(b) $f / \operatorname{div}=200 \mathrm{~Hz}$.

Fig. 10. Waveforms of (a) output current and (b) FFT of output current without any damping.

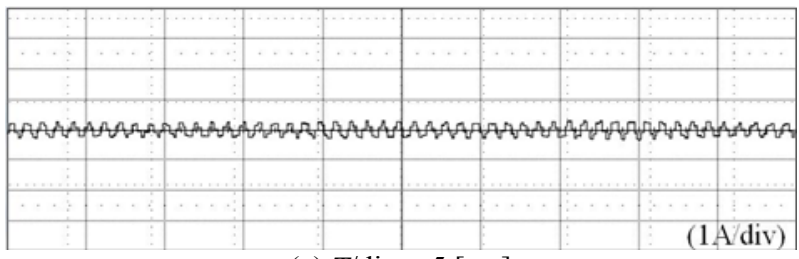

(a) $T / \mathrm{div}=5[\mathrm{~ms}]$

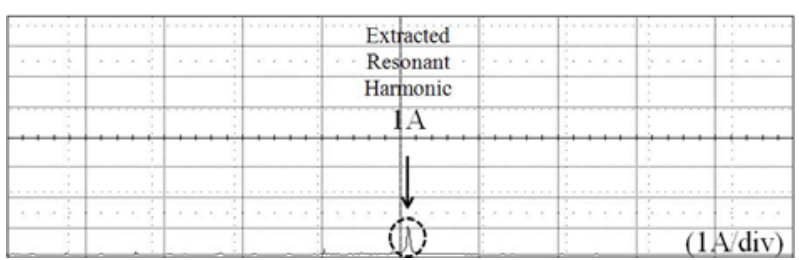

(b) $f / \operatorname{div}=200[\mathrm{~Hz}]$

Fig. 11. Waveforms of (a) extracted resonant signal and (b) FFT of extracted resonant signal with the DFT algorithm. 


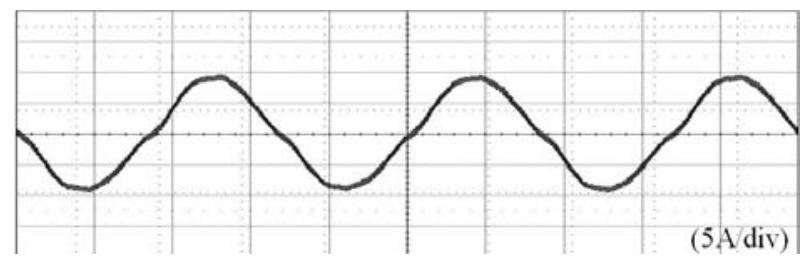

(a) $T / \operatorname{div}=5[\mathrm{~ms}]$

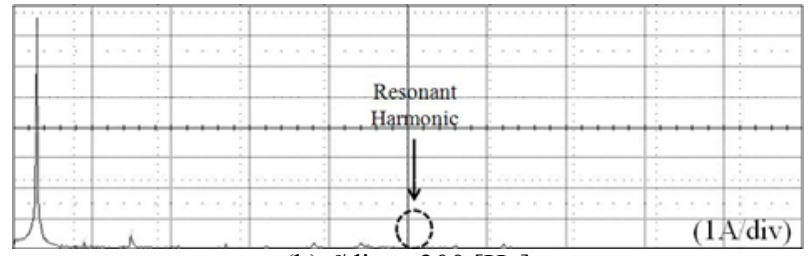

(b) $f / \operatorname{div}=200[\mathrm{~Hz}]$

Fig. 12. Waveforms of (a) output current and (b) FFT with proposed active damping.

the FFT is $1151[\mathrm{~Hz}]$. This magnitude is almost the same as the resonant magnitude in Fig. 10(b). The extracted resonant signal is used to subtract from $i_{\text {grid, }}$, as shown in Fig. 7.

Fig. 12 shows the performance of the proposed active damping method. The resonant ripple of the output currents is eliminated, because the proposed active damping method compensates the resonance. Unlike passive damping, there is no power loss using the DFT.

The superiority of the proposed active damping method is shown in Fig. 12. The DFT can obtain the signal of only the selected frequency. This characteristic enables the compensation of the resonance without any effect on different-order harmonics.

\section{CONCLUSIONS}

This paper proposed an active damping algorithm for wind power systems with an LCL filter. A resonant component was extracted among all of the frequency components by using an DFT filter. The performance of the current controller was improved by using an extracted resonant component. Compared with passive damping, the proposed algorithm does not generate any power loss. The proposed algorithm does not require additional sensors, and it is easily applied without any computational burden. The proposed method was verified by experimental results.

\section{ACKNOWLEDGMENT}

This work was supported by the Human Resources Development of the Korea Institute of Energy Technology Evaluation and Planning (KETEP) grant funded by the Korea government Ministry of Knowledge Economy (No. 20114010203030).

\section{REFERENCES}

[1] K. W. Park and K. B. Lee, "Hardware simulator development for a 3-parallel grid-connected PMSG wind power system," Journal of Power Electronics, Vol. 10, No. 5, pp. 555-562, Sep. 2010.

[2] A. O. D. Tommaso, R. Miceli, G. R. Galluzzo, and M. Trapanese, "Optimum performance of permanent magnet synchronous generators coupled to wind turbines," IEEE PES2007, pp. 1-7, Jun. 2007.

[3] M. Liserre, F. Blaabjerg, and S. Hansen, "Design and control of an LCL-filter-based three-phase active rectifier," IEEE Trans. Ind. Appl., Vol. 41, No. 5, pp. 1284-1285, Sep./Oct. 2005.

[4] I. J. Gabe, V. F. Montagner, and H. Pinheiro, "Design and implementation of a robust current controller for VSI connected to the grid through an LCL filter," IEEE Trans. Power Electron., Vol. 24, No. 6, pp. 1444-1452, Jun. 2009.

[5] D. G. Yoon, H. G. Jeong, and K. B. Lee, "The design of an LCL-filter for the three-parallel operation of a power converter in a wind turbine," IEEE ECCE2010, pp. 1537-1544, 2010.

[6] D. E. Kim and D. C. Lee, "Feedback linearization control of grid-interactive PWM converters with LCL filters," Journal of Power Electronics, Vol. 9, No. 2, pp. 288-299, Mar., 2009.

[7] D. K. Choi and K. B. Lee "Stability improvement of distributed power generation systems with an LCL-filter using gain scheduling based on grid impedance estimations," Journal of Power Electronics, Vol. 11, No. 4, pp. 599-605, Jul. 2011.

[8] H. G. Jung, K. B. Lee, S. Choi, and W. Choi, "Performance improvement of LCL-filter based grid connected-inverters using PQR power transformations," IEEE Trans. Power Electron., Vol. 25, No. 5, pp. 1320-1330, May 2010.

[9] C. P. Dick, S. Richter, M. Rosekeit, J. Rolink, and R. W. De Doncker, "Active damping of LCL resonance with minimum sensor effort by means of a digital infinite impulse response filter," European conference, Power Electronics and Application, pp. 1-8, Sep. 2007.

[10] M. Liserre, A. Dell'Aquila, and F. Blaabjerg, "Genetic algorithm-based design of the active damping for an LCL-filter three-phase active rectifier," IEEE Trans. Power Electron., Vol. 19, No. 1, pp. 76-86, Jan. 2004.

[11] A. Chih-Chiang Hua and B. Chien-Hung Cheng, "Design and implementation of power converters for wind energy conversion system," IEEE IPEC2010, pp 323-328, 2010.

[12] H. S. Bae, J. H. Park, B. H. Cho and G. J. Yu, "New MPPT control strategy for two-stage grid-connected photovoltaic power conditioning system," Journal of Power Electronics, Vol. 7, No. 2, pp. 174-180, Apr. 2007.

[13] T. Ahmedy, K. Nishida, and M. Nakaoka, "Wind power grid integration of an IPMSG using a diode rectifier and a simple MPPT control for grid-side inverters," Journal of Power Electronics, Vol. 10, No. 5, pp. 548-554, Sep. 2010.

[14] M. Felder, J. Mason, and B. Evans, "Efficient dual tone multi-frequency detection using the non-uniform discrete Fourier transform," IEEE Signal processing Letters, Vol. 5, pp. 160-163, Jul. 1998.

[15] E. Jacobsen and R. Lyons, "The sliding DFT," Signal Processing Magazine, IEEE, Vol. 20, No. 2, pp. 74-80, Mar. 2003. 
[16] K. P. Sozanski, "Sliding DFT Control Algorithm for Three-Phase Active Power Filter," APEC '06. Twenty-First Annual IEEE, pp. 1223-1229, Mar. 2006.

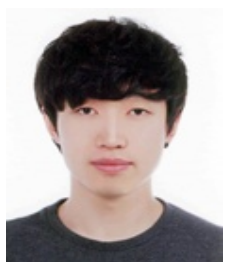

June-Seok Lee was born in Chungnam, Korea, in 1986. He received his B.S. in Electronic Engineering from Ajou University, Suwon, Korea, in 2011. He is currently working toward his M.S. at Ajou University. His research interests include power conversion and grid-connected systems.

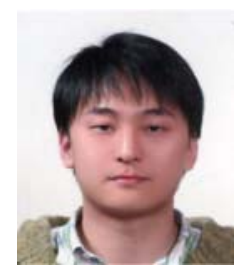

Hae-Gwang Jeong was born in Jeonju, Korea, in 1982. He received his B.S. in Electrical Engineering from Chonbuk National University, Jeonju, Korea, in 2008. $\mathrm{He}$ received his M.S. in Electronic Engineering from Ajou University, Suwon, Korea, in 2010. He is currently working toward his Ph.D. at Ajou University. His research interests include power conversion, grid connected inverters and renewable energy.

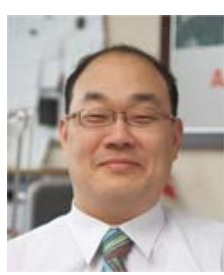

Kyo-Beum Lee was born in Seoul, Korea, in 1972. He received his B.S. and M.S. in Electrical and Electronic Engineering from Ajou University, Suwon, Korea, in 1997 and 1999, respectively. He received his Ph.D. in Electrical Engineering from Korea University, Seoul, Korea, in 2003. From 2003 to 2006, he was with the Institute of Energy Technology, Aalborg University, Aalborg, Denmark. From 2006 to 2007, he was with the Division of Electronics and Information Engineering, Chonbuk National University, Jeonju, Korea. In 2007 he joined the School of Electrical and Computer Engineering, Ajou University, Suwon, Korea. He is an Associated Editor of the IEEE Transactions on Power Electronics, the IEEE Transactions on Industrial Electronics, and the Journal of Power Electronics. His research interests include electric machine drives and renewable power generation. 